\title{
On the Use of Linearized Euler Equations in the Prediction of Jet Noise
}

Reda R. Mankbadi

Lewis Research Center

Cleveland, Ohio

R. Hixon and S.-H. Shih

Institute for Computational Mechanics in Propulsion

Lewis Research Center

Cleveland, Ohio

L.A. Povinelli

Lewis Research Center

Cleveland, Ohio

Prepared for the

33rd Aerospace Sciences Meeting and Exhibit

sponsored by the American Institute of Aeronautics and Astronautics

Reno, Nevada, January 9-12, 1995

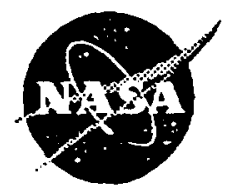

National Aeronautics and

Space Administration

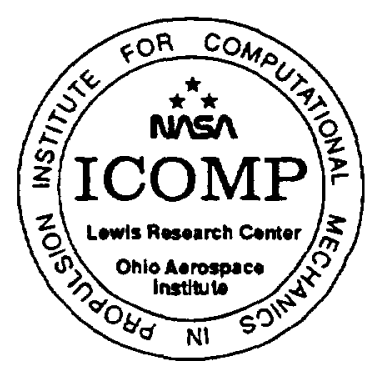





\title{
On the Use of Linearized Euler Equations in the Prediction of Jet Noise
}

\author{
Reda R. Mankbadi* \\ NASA Lewis Research Center \\ Cleveland, $\mathrm{OH} 44135$ \\ R. Hixon**, S.-H. Shih** \\ NASA Lewis Research Center \\ Cleveland, $\mathrm{OH} 44135$ \\ and \\ L. A. Povinelli*** \\ NASA Lewis Research Center \\ Cleveland, $\mathrm{OH} 44135$
}

Institute for Computational Mechanics in Propulsion

\begin{abstract}
Linearized Euler equations are used to simulate supersonic jet noise generation and propagation. Special attention is given to boundary treatment. The resulting solution is stable and nearly free from boundary reflections without the need for artificial dissipation, filtering, or a sponge layer. The computed solution is in good agreement with theory and observation and is much less CPU-intensive as compared to largeeddy simulations.
\end{abstract}

\section{Introduction}

The full, compressible Navier-Stokes equations govern the process of sound generation and propagation to the far field. However, the resolution requirement for high-Reynolds-number turbulent flows makes direct numerical simulation (DNS) impractical due to current computer limitations.

Therefore, Mankbadi et. al. ${ }^{1,2}$ proposed the

* Senior Scientist and Leader, Computational Aeroacoustics; Associate Fellow, AIAA.

* Senior Research Associate, ICOMP; Member, AIAA.

*** Acting Chief, Internal Fluid Mechanics Division; Fellow, AIAA. extension of the large-eddy simulation (LES) approach for use in the prediction of sound generation and propagation. In this approach, the Navier-Stokes equations are filtered into large-scale components, which are calculated directly, and small, unresolved components, which are modeled. The only limitation of an LES approach as opposed to DNS is that sound radiation by the unresolved scales are not accounted for. However, it is believed that the large scales are more efficient than smaller ones for radiating sound. Thus, LES is currently the most accurate approach to jet noise predictions. However, the LES approach is still CPU-intensive, particularly for threedimensional computations of both the near and far fields.

The present work is concerned with exploring the use of the less computerdemanding linearized Euler equations (LEE) for jet noise predictions. The LEE approach neglects both viscosity and nonlinear effects. The viscous effects can be neglected since the large-scale dynamics in free shear flows are essentially inviscid (e.g, Ref. 3). Nonlinearity, however, seems to be important (e.g., Ref. 4).

Yet, much of the physics can be obtained by considering the linear equations. Several attempts have succeeded in studying the physics of jet noise based on a simplified form of the linearized Euler Equations (e.g., Ref. 5-8). The linearized Euler equations 
describe simultaneously both the near field where the sound is generated and the propagation of sound to the far field. As such, the problem of matching the near field to the far field does not arise. The linearized Euler equations fully account for non-parallel flow effects and for the simultaneous presence of non-discrete frequencies.

Special attention is given to the boundary treatment in order to avoid the generation of spurious waves that could render the computed solution entirely unacceptable. Several proposals for boundary treatments are considered, and used where appropriate. The treatment adopted in this work resulted in a stable solution nearly free from reflections without the need to add artificial dissipation, filtering, or sponge layers. The computed solution is found to be in good agreement with theory and observations.

\section{Goveming Equations}

Starting from the full Navier-Stokes equations in conservative form, neglecting viscosity, and linearizing about a mean flow $(U, V)$, the axisymmetric linearized Euler equations may be written in cylindrical coordinates as:

$$
\frac{\partial \tilde{Q}}{\partial t}+\frac{\partial \tilde{F}}{\partial x}+\frac{1}{r} \frac{\partial(r \tilde{G})}{\partial r}=\frac{1}{r} \tilde{S}
$$

where:

$$
\begin{gathered}
\tilde{Q}=\left\{\begin{array}{c}
\tilde{\rho} \\
\tilde{u} \\
\tilde{v} \\
\tilde{e}
\end{array}\right\} \\
\tilde{F}=\left\{\begin{array}{c}
p^{\prime}+2 \bar{u} U-\tilde{\rho} U^{2} \\
\tilde{u} V+\tilde{v} U-\tilde{\rho} U V \\
\left(p^{\prime}+\bar{e}\right) U+(\tilde{u}-\tilde{\rho} U) E
\end{array}\right\}
\end{gathered}
$$

$$
\tilde{G}=\left\{\begin{array}{c}
\tilde{v} \\
\tilde{u} V+\tilde{v} U-\tilde{\rho} U V \\
p^{\prime}+2 \tilde{v} V-\tilde{\rho} V^{2} \\
\left(p^{\prime}+\tilde{e}\right) V+(\tilde{v}-\tilde{\rho} V) E
\end{array}\right\}
$$

and

$$
\tilde{S}=\left\{\begin{array}{l}
0 \\
0 \\
p^{\prime} \\
0
\end{array}\right\}
$$

Here

$$
\begin{gathered}
p^{\prime}=(\gamma-1)\left[\begin{array}{c}
\bar{e}-(\tilde{u} U+\tilde{v} V) \\
+\frac{1}{2} \tilde{\rho}\left(U^{2}+V^{2}\right)
\end{array}\right] \\
P=(\gamma-1) \rho\left[E-\frac{1}{2}\left(U^{2}+V^{2}\right)\right]
\end{gathered}
$$

and

$$
(\tilde{\rho}, \tilde{u}, \tilde{v}, \tilde{e})=\left[\rho^{\prime},(\rho u)^{\prime},(\rho v)^{\prime},(\rho e)^{\prime}\right]
$$

In this notation, $U$ is the axial mean velocity and $\mathrm{V}$ is the radial mean velocity.

Velocities are normalized by the jet exit velocity, time by $D / U_{e}$, density by the mean exit value, and pressure by $\rho_{e} U_{e}{ }^{2}$. Here, D is the nozzle diameter, and the subscript ' $e$ ' denotes the exit value at the centerline.

\section{Mean Flow}

This work uses the analytical functions proposed by Tam and Burton ${ }^{8}$ to fit the experimental data of Troutt and McLaughlin' in the three streamwise regimes of a Mach 2.1 jet: the potential core, transitional, and fully developed regimes.

In the potential core, ranging from $0<x / D$ $<5$, the half-Gaussian (profile I) is used to describe the axial mean flow velocity: 


$$
\begin{array}{ll}
U=1 & \text { for } r<h \\
U=\exp \left[-\ln (2)\left(\frac{r-h(x)}{b(x)}\right)^{2}\right] & \text { for } r>h
\end{array}
$$

In the transitional region, $5<x / D<8$, profile II is used:

$$
\begin{array}{rr}
U=U_{c}(x) & \text { for } \mathrm{r}<\mathrm{h} \\
U=U_{c}(x) \exp \left[-\ln (2)\left(\frac{r-h(x)}{b(x)}\right)^{2}\right] & \\
\text { for } \mathrm{r}>\mathrm{h}
\end{array}
$$

In the fully developed regime, $x / D>8$, profile III is used:

$$
U=U_{c}(x) \exp \left[-\ln (2)\left(\frac{r}{b(x)}\right)^{2}\right]
$$

where $b(x)$ is the half-width of the annular mixing layer and is fitted to the experimental data. The radius of the uniform core $h(x)$ and the centerline velocity $U_{c}(x)$ are related to $b(x)$ through the conservation of momentum:

$$
\int_{0}^{\infty} \rho U^{2} r d r=\frac{1}{2}
$$

For profile $I, U_{c}=1$, and hence equation (12) is used to obtain $h(x)$ in terms of $b(x)$. For profile III, equation (12) is used to obtain $U_{c}(x)$ in terms of $b(x)$. For profile II, $b(x)$ and $h(x)$ are obtained by using a cubic spline fit that matches the values of $b(x)$ and its derivative to that of profiles I and III and likewise for $h(x)$.

Invoking the boundary-layer-type approximation to the mean flow equations shows that the mean pressure can be taken to be uniform in the jet. Under such assumptions, one can show that the continuity equation for the mean flow reduces to that of the incompressible flow, which is used to obtain the radial flow velocity $V(r)$ as:

$$
V(r)=-\frac{1}{r} \int_{0}^{\infty} \frac{\partial U}{\partial x} r d r
$$

The above profiles are used to describe the mean flow up to maximum radius $r_{\max }=h+$ $3 b$. For $r>r_{\max }$

$$
\begin{aligned}
& U=0 \\
& V=\frac{V_{\infty}}{r}
\end{aligned}
$$

where $V_{\infty}$ is the uniform radial velocity in the outer regime. By assuming the total temperature to be uniform, the relation between the static temperature and the axial mean flow velocity is obtained. The equation of state is then used to obtain the mean density in terms of the static temperature.

The computational grid for this problem extends axially from $x / D=2.5$ to $x / D=35$, using 196 equally spaced points ( 25 points per wavelength). Due to the steep meanflow gradients encountered at the jet exit, the computational grid was begun at an axial distance $x / D=2.5$ from the actual jet exit.

In the radial direction, the grid begins just above the centerline $(r / D=0.005)$ and extends to $r / D=16$, with a total of 381 points. The grid is uniform from the centerline to $r / D=1$, with a spacing of $\Delta r / D=0.01$. At this point, the grid is stretched geometrically by a factor of 1.01 , until the radial spacing is equal to the axial spacing. After this point, the grid is uniform again to the outer radial boundary.

\section{Numerical Algorithm}

The code is a modified split MacCormack solver, which is second order accurate in time and fourth order accurate in space. This extension of the MacCormack scheme is known as the 2-4 scheme, and was developed by Gottleib and Turkel ${ }^{10}$. This scheme has been used successfully on a wide range of fluid and aeroacoustics problems ${ }^{11-}$ 24. Sankar, Reddy, and Hariharan ${ }^{25}$ have 
evaluated this scheme for aeroacoustics applications. The solution procedure is as follows:

In the present code, the operator is split into separate radial and axial contributions:

$$
q^{n+2}=L_{x} L_{r} L_{r} L_{x} q^{n}
$$

Each operator consists of a predictor and a corrector step. Each step uses one-sided differencing:

Predictor:

$$
q^{n+\frac{1}{2}}=q^{n}-\frac{\Delta t}{6 \Delta x}\left(7 F_{i}-8 F_{i-1}+F_{i-2}\right)^{n}
$$

Corrector:

$$
q^{n+1}=\frac{1}{2}\left(\begin{array}{l}
q^{n}+q^{n+\frac{1}{2}} \\
+\frac{\Delta t}{6 \Delta x}\left(7 F_{i}-8 F_{i+1}+F_{i+2}\right)^{n+\frac{1}{2}}
\end{array}\right)
$$

and likewise for the radial direction. The sweep directions are reversed between operators to avoid biasing. At the computational boundaries, flux quantities outside the boundaries are needed to compute the spatial derivatives, and these can be obtained using third-order extrapolation based on data from the interior of the domain.

\section{Boundary Treatment}

Special attention is given herein to boundary treatment in order to avoid nonphysical oscillations that can render the computed oscillating field unacceptable. Several boundary treatments were considered. ${ }^{26.27}$ The boundary treatments discussed below were found to be stable, non-reflecting, and suitable for the present jet computations.

\subsection{Inflow Boundary Conditions}

At the inflow boundary $(x=0)$, the radial boundary is split into hydrodynamic disturbance and radiation regimes, which are treated differently as outlined below.

5.1.1 Inflow Disturbance At the inflow boundary, a small disturbance is introduced. This disturbance is assumed to be mainly hydrodynamic in nature, and is specified from the centerline to $r / D=2$.

To a first approximation, the inflow disturbance is assumed to be small such that the linear stability theory applies. A normal mode decomposition for the disturbance is assumed in the form:

$$
\begin{gathered}
{\left[u^{\prime}, v^{\prime}, p^{\prime}, \rho^{\prime}\right]=} \\
\operatorname{Re}[\tilde{u}(r), \tilde{v}(r), \tilde{p}(r), \tilde{\rho}(r)] \exp \{i(\alpha x-\omega t)\}
\end{gathered}
$$

The governing equations reduce to the OrrSommerfeld equation, which is solved to obtain the complex wave number $\alpha$ as the eigenvalue corresponding to the frequency $\omega$ and the radial functions $(\sim)$ as the corresponding eigenfunctions. The mean flow discussed in Section 3 is used in solving the Orr-Sommerfeld equation.

This solution extends to $r / D=1$. A curve is fitted to smoothly set the disturbance to zero by $r / D=2$.

The effect of the inflow disturbance is added to the computed flow variables at the inflow boundary at each time step:

$$
\left(Q_{t}\right)_{\text {boundary }}=\left(Q_{t}\right)_{\text {compuled }}+\left(Q_{t}\right)_{\text {disturbance }}
$$

\subsubsection{Hydrodynamic Disturbance Regime} In the hydrodynamic disturbance regime $(\mathrm{r} / \mathrm{D}<2)$, the Thompson inflow boundary condition ${ }^{28,29}$ is used. In the Thompson analysis, the axial operator is decomposed into four 1-D characteristics. At a subsonic inflow boundary, three of these characteristics are incoming, and are set to zero for a non-reflective boundary condition, while the fourth characteristic is outgoing and is computed from the flow solution: 


$$
\begin{aligned}
& p_{t}^{\prime}+\overline{\rho c} u_{t}^{\prime}=0 \\
& \dot{p_{t}^{\prime}}+\bar{c}^{2} \rho_{t}^{\prime}=0 \\
& \bar{\rho} \bar{c} v_{t}^{\prime}=0 \\
& \dot{p_{t}}+\overline{\rho c} \overline{u_{t}^{\prime}}=R_{4}
\end{aligned}
$$

The four characteristic equations are then solved together to obtain the time derivatives of the variables at the inflow boundary. For a supersonic inflow, all characteristics are incoming, and are all set to zero.

Due to the specified disturbance at the inflow boundary, the Thompson inflow boundary condition exhibited a problem in which some disturbances were convected in a radial direction and remained on the boundary for the rest of the computation. To alleviate this, the mean radial velocity was set to zero on the boundary, and smoothly raised to the proper value by $x / D$ $=5.7$.

5.1.3 Radiation regime In the radiation regime ( $r / D>2)$, the conventional acoustic radiation condition applies:

$$
Q_{t}^{\prime}=-V(\theta)\left[\frac{x}{R} Q^{\prime}{ }_{x}+\frac{r}{R} Q_{r}^{\prime}+\frac{Q^{\prime}}{R}\right]
$$

where:

$$
\begin{aligned}
& Q^{\prime}=\left\{\begin{array}{l}
\rho^{\prime} \\
u^{\prime} \\
v^{\prime} \\
p^{\prime}
\end{array}\right\} \\
& R=\sqrt{x^{2}+r^{2}} \\
& V(\theta)=\bar{c}\left[\frac{x}{R} M+\sqrt{1-\left(\frac{r}{R} M\right)^{2}}\right]
\end{aligned}
$$

and $M$ is the local Mach number. The spatial derivatives which appear in Eq. (21) are evaluated in an identical manner as the inner flow derivatives.

\subsection{Outflow Boundary Conditions}

The outflow treatment is based on the asymptotic analysis of the linearized equations as given by Tam and Webb. ${ }^{30}$ The pressure condition is the same as that obtained by Bayliss and Turkel ${ }^{31}$, Enquest and Majda ${ }^{32}$, and Hariharan and Hagstrom $^{33}$, namely:

$$
p_{t}^{\prime}=-V(\theta)\left[\frac{x}{R} p^{\prime}{ }_{x}+\frac{r}{R} p^{\prime}{ }_{r}+\frac{p^{\prime}}{R}\right]
$$

However, for updating the rest of the primitive variables, Tam and Webb have shown that the momentum and continuity equations should be used to account for the presence of entropy and vorticity waves at the outflow boundary. The spatial differencing used in the inner code is employed to evaluate the derivatives which appear in Eq. (23).

For the outflow regime of large radius and a local Mach number less than 0.01, the outflow condition is replaced by the radiation condition of Section 5.1.3.

It must be noted that the Tam and Webb outflow boundary condition is formulated with an assumption that the mean flow is uniform, which is not true for the jet outflow. However, the results given by this boundary condition were quite good, with very little reflection.

\subsection{Quter Radial Boundary Condition}

At the outer radial boundary $\left(r=r_{\max }, 0<x\right.$ $\left.<x_{\max }\right)$, the radiation boundary condition of Section 5.1.3 is used.

\subsection{Centerline Treatment}

For an axisymmetric problem, the boundary condition at $r=0$ can be stated as:

$$
\frac{\partial}{\partial r}\left\{\begin{array}{l}
\rho^{\prime} \\
u^{\prime} \\
p^{\prime}
\end{array}\right\}=0
$$




$$
v^{\prime}=0
$$

To implement this boundary condition numerically, the fluxes are projected to ghost points across the centerline in an appropriate manner. The centerline treatment for a non-axisymmetric case is not obvious, and is addressed in a separate paper by Shih, et. al. ${ }^{34}$

\section{Results}

Results are presented for the axisymmetric flow and acoustic field of a supersonic jet (M $=2.1$ ), unheated with a uniform stagnation temperature of $270^{\circ} \mathrm{Kelvin}$. The Reynolds number of the mean flow is 70,000 , and the jet is excited at a Strouhal number of 0.2 . This case was tested experimentally by Troutt and McLaughlin ${ }^{9}$, and theoretically by Tam and Burton. ${ }^{8}$

\subsection{The Global Field}

The global field of the jet is shown in Figures 1-7. Figure 1 shows the oscillating pressure field with the appropriate boundary treatment indicated on the figure. The solution is stable and clean from boundary reflections. Close to the axis, we note the oscillatory nature of the sound source, which will be shown in more detail in figures 8-12. The sound source radiates sound that seems to peak at an angle influenced by the streamwise position where the disturbance reaches a maximum.

Figure 2 shows the instantaneous distribution of $u^{\prime}, v^{\prime}$, dilation, and vorticity. We note that the vorticity is confined to the near field, showing that the calculated outer field is a truly irrotational acoustic field.

Figure 3 shows contours of the maximum and root-mean-square values of the pressure oscillation. The field resembles that of a quadrupole with preferred forward emission.

The directivity of sound is shown in Figure 4. The peak occurs around 26 degrees, which is in close agreement with the theoretical prediction of Tam and Burton, which were obtained via matched asymptotic expansion. The experimental results of Troutt and McLaughlin, in which an initially laminar jet was excited with a disturbance at $\mathrm{St}=0.2$. As pointed out by Troutt and McLaughlin, the measured sound source was found to be composed of axisymmetric and helical modes, with the latter more dominant than the former. The results presented here are only for the axisymmetric mode, but still the qualitative agreement between the calculation and experiment is evident.

Figure 5 shows the root-mean-square pressure disturbance along the $r / D=8.0$ line, compared to the experiment of Troutt and McLaughlin. The results show a qualitative similarity, with an axial shift of the maximum disturbance.

The radial decay of the pressure and axial velocity disturbance field is shown in figures 6 and 7 , indicating a $1 / R$ decay, as expected for the acoustic field.

Figure 8 shows the spectra of the sound pressure field at $x / D=22.3$ and $r / D=11.8$. The dominant frequency is that of the input disturbance.

Figure 9 shows the sound pressure level distribution in the far field for the present work, the axisymmetric mode of Tam and Burton, and the experimental measurements of Troutt and McLaughlin. It is seen that the graphs are all qualitatively similar, but the present results show an upstream axial shift of the lobes when compared to Tam and Burton's previous work. The lobes are shifted a distance of $x / D=2.5$ from Tam and Burton's analytical calculation, and $x / D=1.5$ from Troutt and McLauglin's experimental results.

\subsection{The Near Field}

The near field is shown in more detail in figures 10-14.

Instantaneous distributions of the pressure disturbance in the shear layer are shown in 
Figure 10. The effects of the boundary conditions on the pressure disturbance are evident in this figure; reflections from the front and rear boundaries are in evidence.

Figure 11 shows instantaneous distributions of the axial velocity disturbance along the $r / D=.5$ line for several time levels. The boundary conditions seem to have much less effect on the axial velocity disturbances.

Figure 12 shows the spectra of the sound pressure field at several points in the near field. It is again seen that the dominant frequency is that of the input disturbance. However, some very high frequency noise is seen at the downstream points, and some middle frequency noise is seen at the point near the outflow boundary.

Figure 13 shows the root-mean-square pressure disturbance distribution in the shear layer. There are outflow boundary reflections evident in the downstream portion.

Figure 14 compares the axial momentum disturbance growth in the shear layer with the experimental results of Troutt and McLaughlin. The maximum value of the graphs are matched, and the results agree well.

\section{Conclusions}

A linearized Euler equation approach is used to compute the sound propagation in a supersonic jet. The boundary treatment used provides a stable solution with very little reflection from the boundary. The scheme is fast, and can be used for extension to three-dimensional flow fields and for parametric studies. The computed results were found to be in good agreement with the matched asymptotic solution of Tam and Burton, and in qualitative agreement with the experimental results of Troutt and McLaughlin.

\section{References}

1) Mankbadi, R. R., Hayder, M. E., and Povinelli, L. A., 'Structure of Supersonic Jet Flow and Its
Radiated Sound', AIAA Journal, Vol. 32, No. 5, May 1994, pp. 897906.

2) Mankbadi, R. R., Shih, S.-H., Hixon, R., and Povinelli, L. A., 'Direct Computation of Acoustic and Flow Field of a Supersonic Jet Using Large-Eddy Simulation', AIAA Paper 95-0680, Reno, NV, Jan. 1995.

3) Mankbadi, R. R. 'Dynamics and Control of Coherent Structure in Turbulent Jets', Appl. Mech. Reviews, 45, 1992, p. 219-248.

4) Laufer, J. and Yen, T. C., 'Noise Generation by a Low Mach Number Jet', J. Fluid Mech., Vol. 62, 1983, p. 437-464.

5) Crighton, D. G. and Huerre, P., 'Shearlayer Pressure Fluctuations and Superdirective Acoustic Sources', J. Fluid Mech., Vol. 220, 1990, p. 355368.

6) Liu, J. T. C, 'Developing Large-Scale Wavelike Eddies and the Near Jet Noise Field', J. Fluid Mech., Vol. 62, 1974, p. 437-464.

7) Tam, C. K. W., 'Supersonic Jet Noise', Annu. Rev. Fluid Mech., 27, 1995, p. 17-43.

8) Tam, C.K.W., and Burton, D. E., 'Sound Generated by Instability Waves of Supersonic Flows, Part 2: Axisymmetric Jets', J. Fluid Mech., Vol. 138, 1984, p. 273-295.

9) Troutt, T. R., and McLaughlin, D. K., 'Experiments on the Flow and Acoustic Properties of a ModerateReynolds-Number Supersonic Jet', J. Fluid Mech., Vol. 116, 1982, p. 123156.

10) Gottlieb, D., and Turkel, E., 'Dissipative Two-Four Method for Time Dependent Problems', Mathematics of Computation, Vol. 30, No. 136, 1976, pp. 703-723. 
11) Bayliss, A. and Maestrello, L., 'Simulation of Instabilities and Sound Radiation in a Jet', AIAA Journal, Vol. 19, 1981, p. 835-841.

12) Maestrello, L., Bayliss, A., and Turkel, E., 'On the Interaction of a Sound Pulse with the Shear Layer of an Axisymmetric Jet', Journal of Sound and Vibration', Vol. 74, 1981, p. 281-301.

13) Bayliss, A., and Maestrello, L., 'On the Interaction of a Sound Pulse with the Shear Layer of an Axisymmetric Jet II: Heated Jets', Journal of Sound and Vibration, Vol. 86, 1982, p. 395-409.

14) Maestrello, L., and Bayliss, A., 'Flowfield and Far Field Acoustic Amplification Properties of Heated and Unheated Jets', AIAA Journal, Vol. 20, 1982, p. 1539-1546.

15) Bayliss, A., Parikh, P., Maestrello, L., and Turkel, E., 'A Fourth Order Scheme for the Unsteady Compressible Navier-Stokes Equations', AIAA Paper 85-1694, 1985.

16) Bayliss, A., Maestrello, L., Parikh, P., and Turkel, E., 'Numerical Simulation of Boundary Layer Excitation by Surface Heating/Cooling', AIAA Journal, Vol. 24, 1986, p. 1095-1101.

17) Bayliss, A., Maestrello, L., and Turkel, E., 'On the Interaction of a Sound Pulse with the Shear Layer of an Axisymmetric Jet III: Non-Linear Effects', Journal of Sound and Vibration, Vol. 107, 1986, p. 167175.

18) Maestrello, L., Bayliss, A., Parikh, P., and Turkel, E., 'Instability and Sound Emission from a Flow over a Curved Surface', Journal of Vibration, Stress, and Reliability in Design, Vol. 110, 1988, p.538-544.
19) Maestrello, L., Bayliss, A., and Krishnan, R., 'On the Interaction Between First- and Second-Mode Waves in a Supersonic Boundary Layer', Physics of Fluids A, Vol. 3, 1991, p. 3014-3020.

20) Frendi, A., Maestrello, L., and Bayliss, A., 'Coupling Between a Supersonic Boundary Layer and a Flexible Surface', AIAA Journal, Vol. 31, 1993, p. 708-713.

21) Frendi, A., Maestrello, L., and Bayliss, A., 'Coupling Between Plate Vibration and Acoustic Radiation', Journal of Sound and Vibration, Vol. 177, 1994, p. 207-226.

22) McGreevy, J.L., Bayliss, A., and Maestrello, L., 'On the Interaction of Jet Noise with a Nearby Panel Assembly', to appear in AIAA Journal.

23) Farouk, B., Oran, E. S., and Kailasanath, K., 'Numerical Simulations of the Structure of Supersonic Shear Layers', Physics of Fluids A, Vol. 3, No. 11, 1991, pp. 2786-2798.

24) Ragab, S. A., and Sheen, S., 'The Nonlinear Development of Supersonic Instability Waves in a Mixing Layer', Physics of Fluids $A$, Vol. 4, No. 3, 1991 , pp. 553-566.

25) Sankar, L. N., Reddy, N. N., and Hariharan, N., 'A Comparative Study of Numerical Schemes for Aero-Acoustic Applications', FEDVol. 147, Computational Aero- and Hydro-Acoustics, ASME 1993, p. 3540.

26) Hixon, R., Shih, S.-H., and Mankbadi, R. R., 'Evaluation of Boundary Conditions for Computational Aeroacoustics', AlAA Paper 950160, Reno, NV, Jan. 1995. 
27) Scott, J. N., Mankbadi, R. R., Hayder, M. E., and Hariharan, S. I., 'Outflow Boundary Conditions for the Computational Analysis of Jet Noise', AIAA Paper 93-4366, 1993.

28) Thompson, K.W, 'Time-Dependent Boundary Conditions for Hyperbolic Systems', $J$. Computational Physics, Vol. 68, Jan. 1987, p. 1-24.

29) Thompson, K.W, 'Time-Dependent Boundary Conditions for Hyperbolic Systems, II', $J$. Computational Physics, Vol. 89, 1990 , p. $439-461$.

30) Tam, C. K. W. and Webb, J. C., 'Dispersion-Relation-Preserving Finite Difference Schemes for Computational Acoustics', J. Computational Physics, Vol. 107, 1993, pp. 262-281.

31) Bayliss, A., and Turkel, E., 'Far Field Boundary Conditions for Compressible Flows', $J$. Computational Physics, Vol. 48, 1982, p. 182-199.

32) Enquist, B., and Majda, A., 'Radiation Boundary Conditions for Acoustic and Elastic Wave Calculations', Communications on Pure and Applied Mathematics, Vol. 32, No. 3, 1979, pp. 313-357.

33) Hagstrom, T., and Haraharan, S. I., 'Far Field Expansion for Anisotropic Wave Equations', Computational Acoustics, Vol. 2, 1990.

34) Shih, S.-H., Hixon, R., and Mankbadi, R. R., 'Three-Dimensional Structure in a Supersonic Jet: Behavior Near Centerline', AIAA Paper 95-0681, Reno, NV, Jan. 1995.

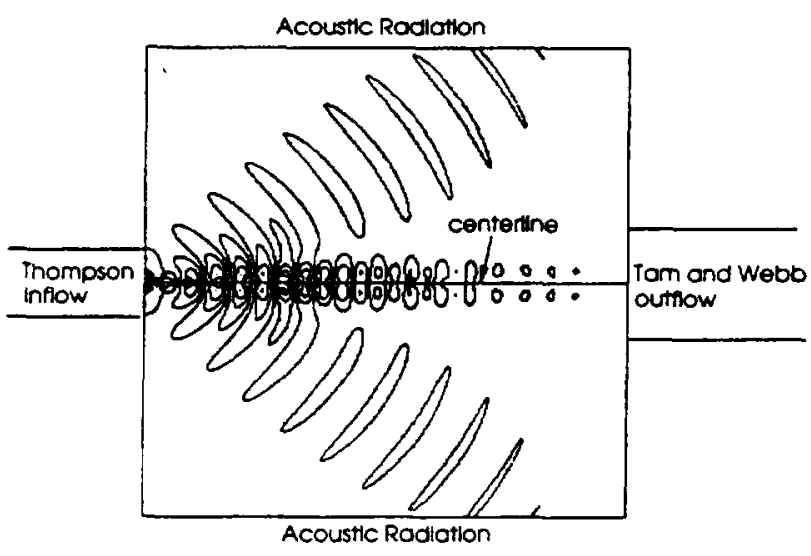

Figure 1

Illustration of Oscillating Pressure Field and Boundary Conditions

$\left(M_{\text {jet }}=2.1 ; S t=0.2\right.$; axisymmetric mode $)$

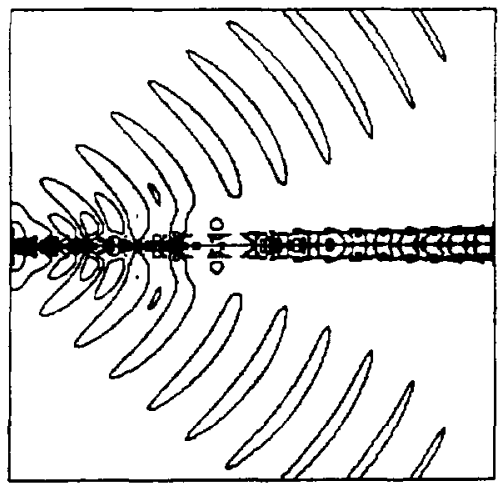

Figure 2a

Instantaneous Distribution of the Axial Velocity Disturbance Field

$\left(M_{\text {jet }}=2.1 ; \mathrm{St}=0.2\right.$; axisymmetric mode $)$

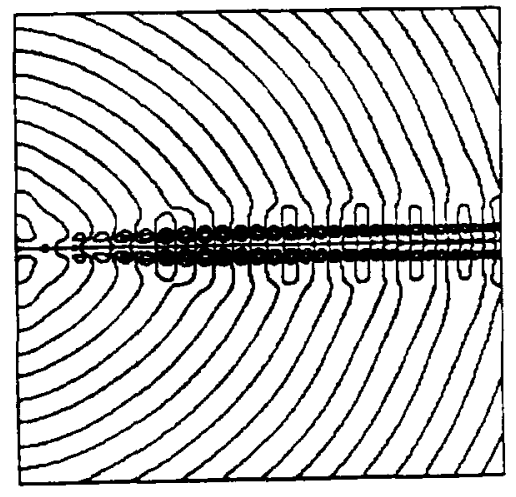

Figure $\mathbf{2 b}$

Instantaneous Distribution of the Radial Velocity Disturbance Field

$\left(M_{\text {jet }}=2.1 ; S t=0.2 ;\right.$ axisymmetric mode $)$ 


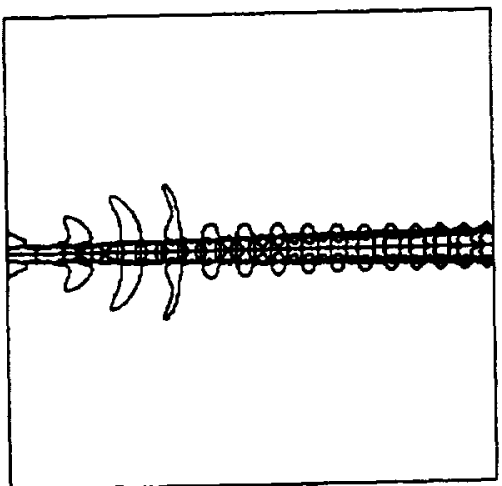

Figure 2c

Instantaneous Distribution of the Disturbance Velocity Dilatation $\left(M_{\text {jet }}=2.1 ; \mathrm{St}=0.2 ;\right.$ axisymmetric mode $)$

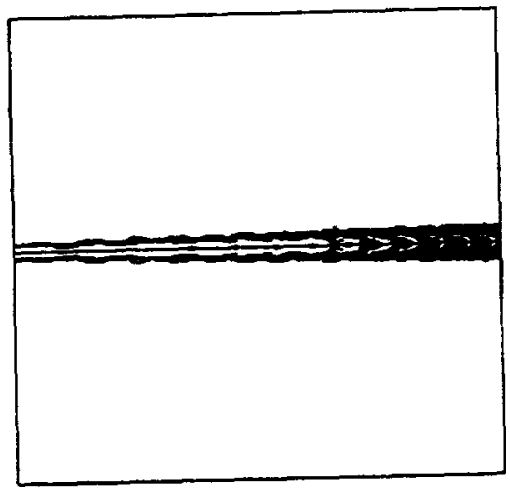

Figure 2d

Instantaneous Distribution of the Disturbance Vorticity $\left(M_{\text {jet }}=2.1 ; S t=0.2 ;\right.$ axisymmetric mode $)$

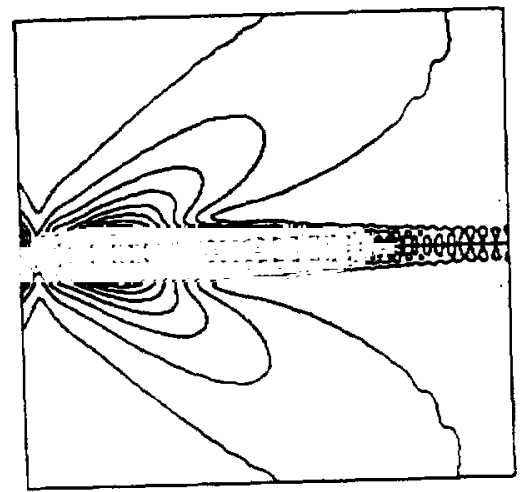

Figure 3a

Maximum Values of the Pressure Oscillation $\left(\mathrm{M}_{\mathrm{jet}}=2.1 ; \mathrm{St}=0.2 ;\right.$ axisymmetric mode $)$

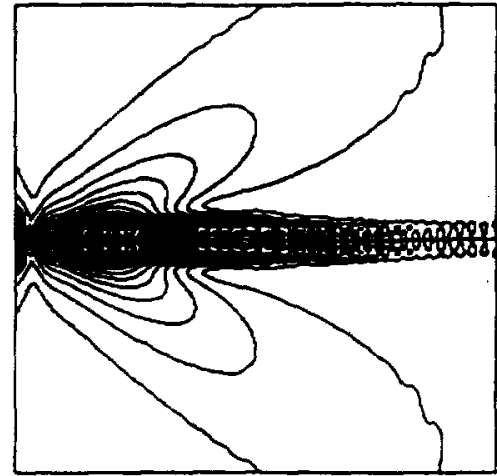

Figure 3b

Root-Mean-Square Values of the Pressure Oscillation

$\left(\mathrm{M}_{\mathrm{jet}}=2.1 ; \mathrm{St}=0.2 ;\right.$ axisymmetric mode $)$

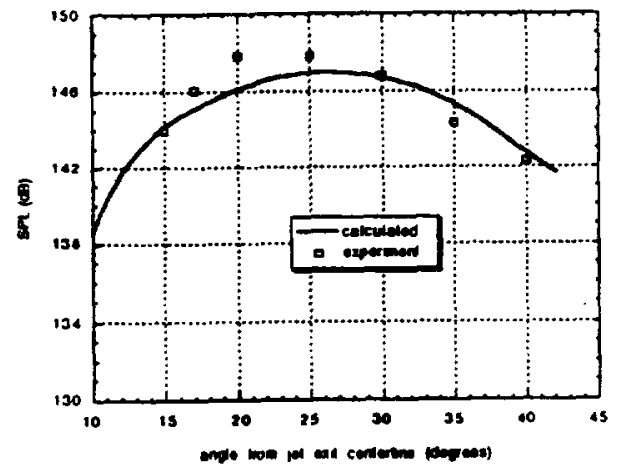

Figure 4

Directivity of Jet Noise

$\left(M_{\text {jet }}=2.1 ; S t=0.2 ; R / D=24\right)$

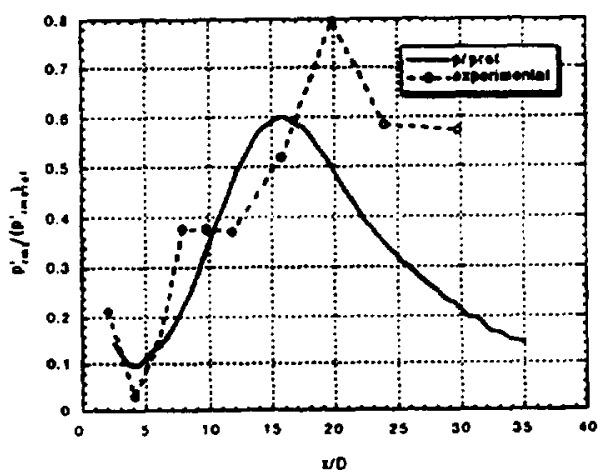

Figure 5

Comparison of SPL levels along the $r / D=8.0$ line $\left(M_{\text {jet }}=2.1 ; S t=0.2 ;\right.$ axisymmetric mode $)$ 


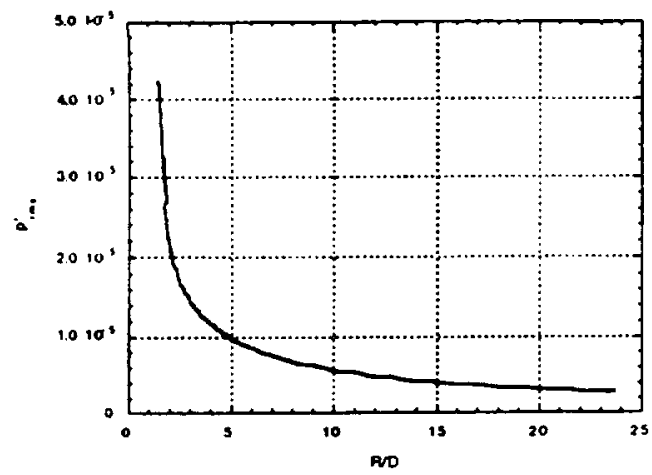

Figure 6

Radial Decay of the Pressure Disturbance in the Far Field

$\left(\mathrm{M}_{\mathrm{jet}}=2.1 ; \mathrm{St}=0.2\right.$; axisymmetric mode $)$

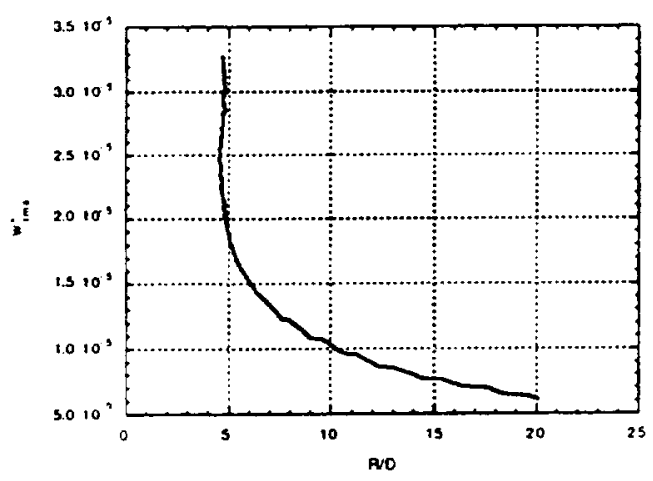

Figure 7

Radial Decay of the Axial Velocity

Disturbance in the Far Field

( $\mathrm{M}_{\mathrm{jet}}=2.1 ; \mathrm{St}=0.2 ;$ axisymmetric mode)

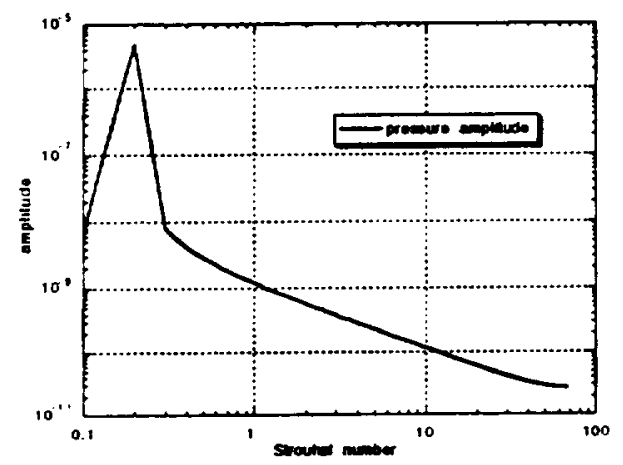

Figure 8

Far Field Pressure Spectra at $(20,23.5)$ $\left(\mathrm{M}_{\text {jet }}=2.1 ; \mathrm{St}=0.2\right.$; axisymmetric mode $)$

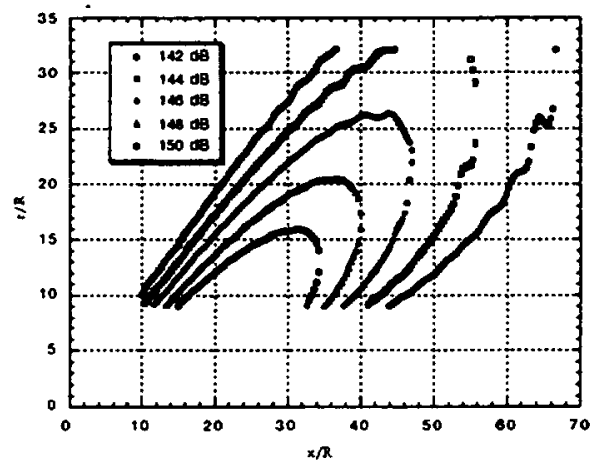

Figure 9a

SPL contours for present calculation $\left(M_{\text {jet }}=2.1 ; S t=0.2\right.$; axisymmetric mode $)$

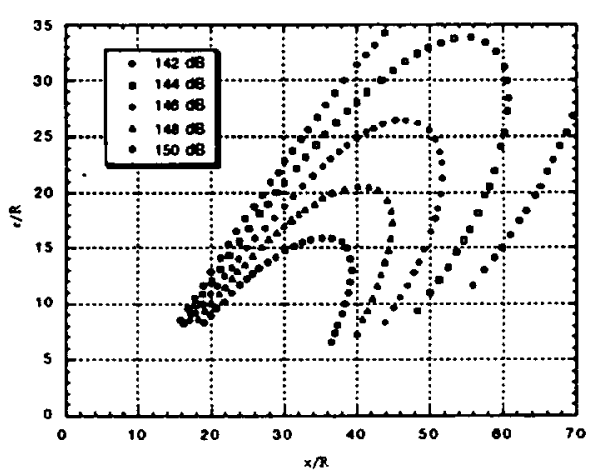

Figure $9 b$

SPL contours for Tam and Burton calculation

$\left(M_{\text {jet }}=2.1 ; S t=0.2\right.$; axisymmetric mode $)$

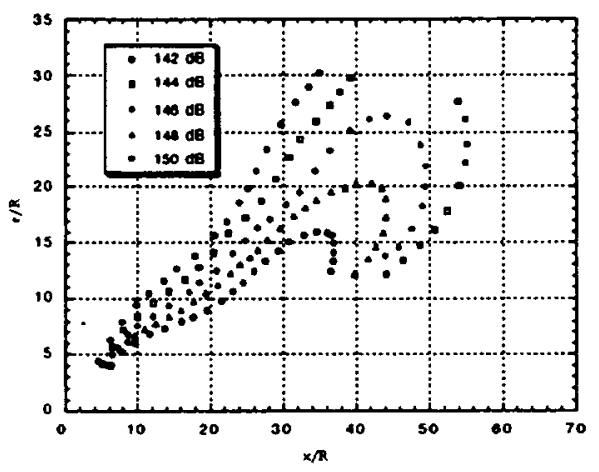

Figure 9c

SPL contours for Troutt and McLaughlin experiment

$\left(M_{\text {jet }}=2.1 ; S t=0.2\right)$ 


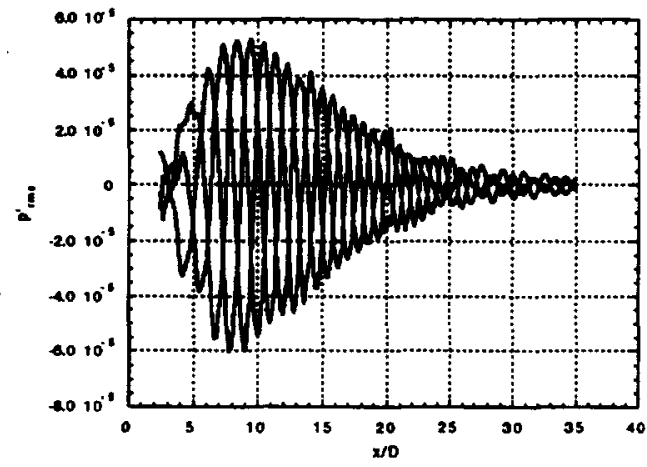

Figure 10

Instantaneous Pressure Disturbance along the $r / D=0.5$ line $\left(M_{\text {jet }}=2.1 ; \mathrm{St}=0.2 ;\right.$ axisymmetric mode $)$

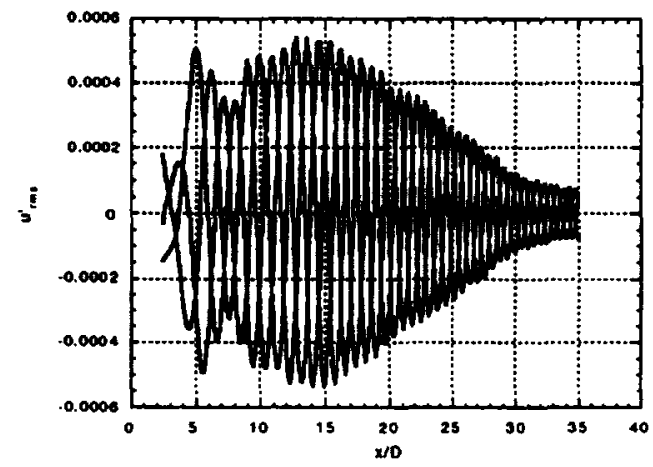

Figure 11

Instantaneous Axial Velocity Disturbance along the $r / D=0.5$ line $\left(M_{\text {jet }}=2.1 ; \mathrm{St}=0.2 ;\right.$ axisymmetric mode $)$

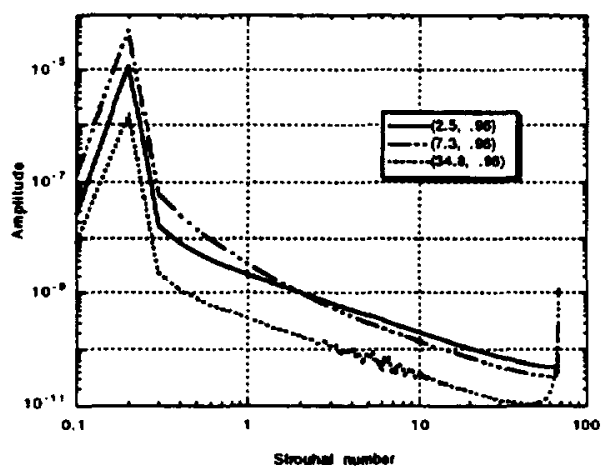

Figure 12

Near Field Pressure Spectra in the Shear Layer

$\left(M_{\text {jet }}=2.1 ; S t=0.2 ;\right.$ axisymmetric mode $)$

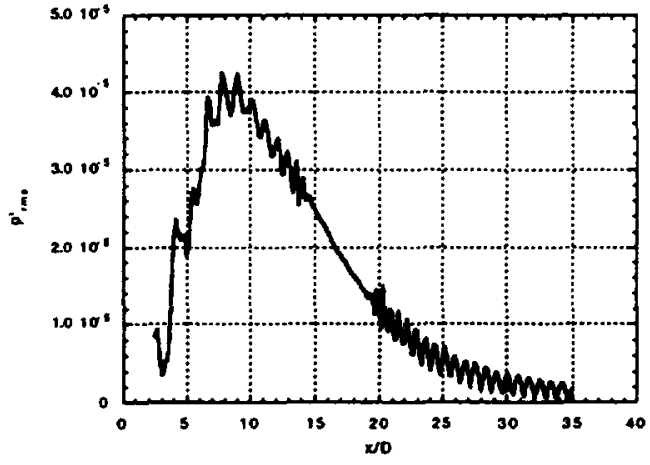

Figure 13

RMS Pressure Disturbance in the Shear Layer $(r / D=0.5)$

$\left(M_{\text {jet }}=2.1 ; S t=0.2 ;\right.$ axisymmetric mode $)$

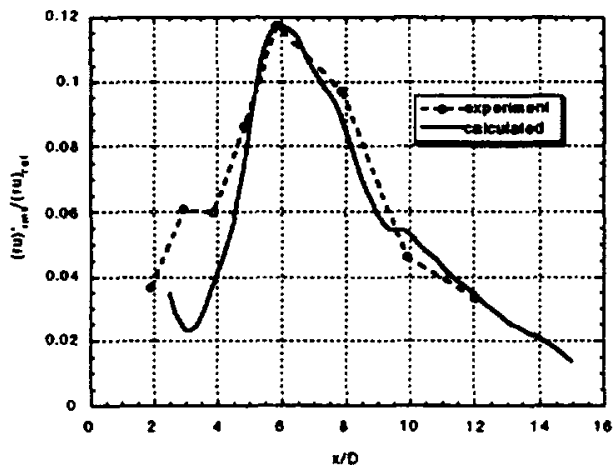

Figure 14

Distribution of the RMS axial momentum disturbance in the shear layer

$\left(M_{\text {jet }}=2.1 ; \mathrm{St}=0.2 ;\right.$ axisymmetric mode $)$ 


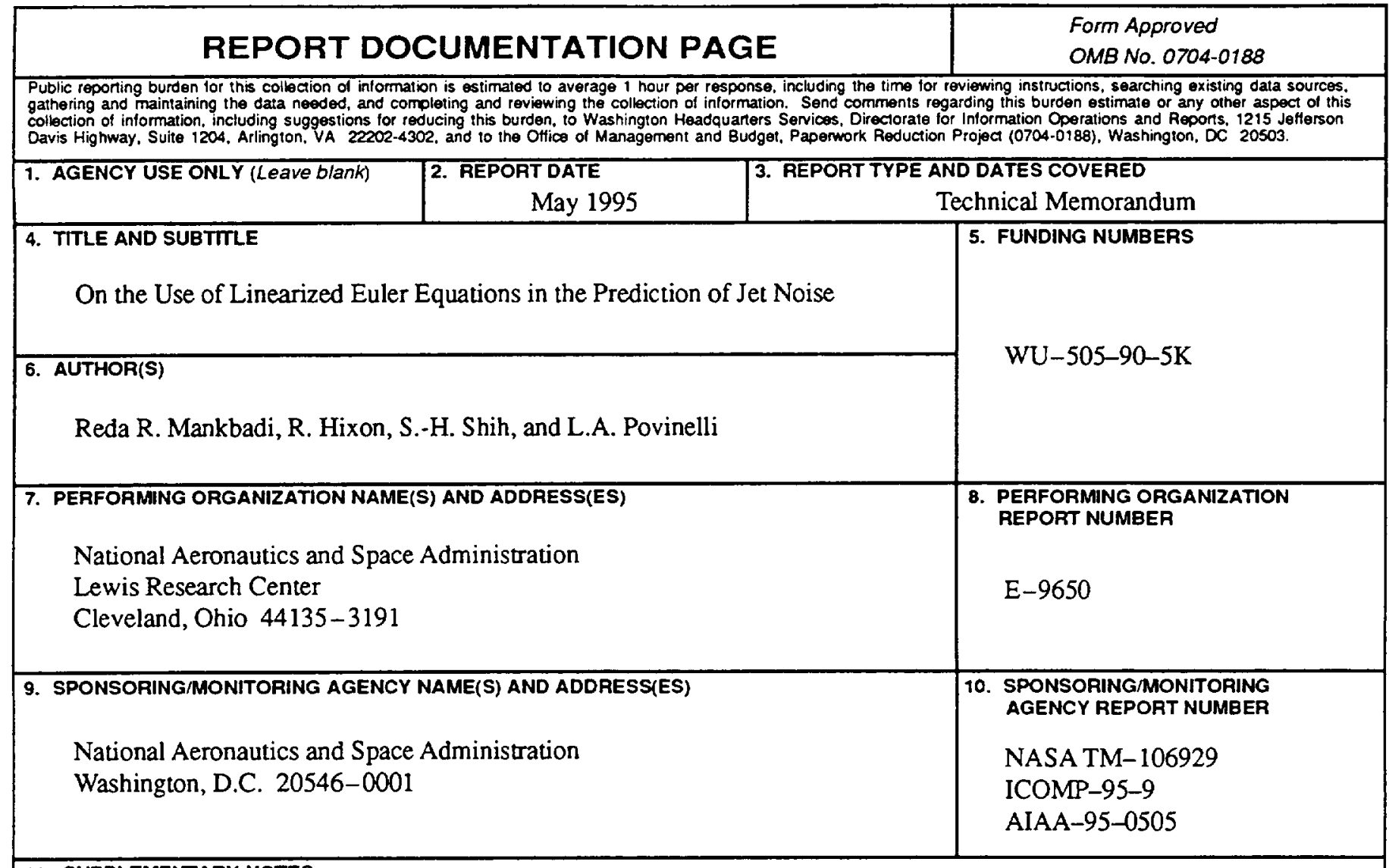

\section{SUPPLEMENTARY NOTES}

Prepared for the 33rd Aerospace Sciences Meeting and Exhibit sponsored by the American Institute of Aeronautics and Astronautics, Reno, Nevada, January 9-12, 1995. Reda R. Mankbadi and L.A. Povinelli, NASA Lewis Research Center; R. Hixon and S.-H. Shih, Institute for Computational Mechanics in Propulsion, NASA Lewis Research Center (work funded under NASA Cooperative Agreement NCC3-370). ICOMP Program Director, Louis A. Povinelli, organization code $2600,(216) 433-5818$.

\begin{tabular}{|l|l} 
12a. DISTRIBUTIONVAVAILABILTTY STATEMENT & 12b. DISTRIBUTION CODE
\end{tabular}

Unclassified - Unlimited

Subject Category 34

This publication is available from the NASA Center for Aerospace Information, (301) 621-0390.

13. ABSTRACT (Maximum 200 words)

Linearized Euler equations are used to simulate supersonic jet noise generation and propagation. Special attention is given to boundary treatment. The resulting solution is stable and nearly free from boundary reflections without the need. for artificial dissipation, filtering, or a sponge layer. The computed solution is in good agreement with theory and observation and is much less CPU-intensive as compared to large-eddy simulations.

14. SUBJECT TERMS

Computational aeroacoustics; Jet noise; Linearized Euler equations; Boundary conditions 15. NUMBER OF PAGES 14
16. PAICE CODE $\mathrm{AO} 3$

17. SECURTY CLASSIFICATION OF REPORT Unclassified
18. SECURTY CLASSIFICATION OF THIS PAGE Unclassified
19. SECURITY CLASSIFICATION OF ABSTRACT Unclassified 




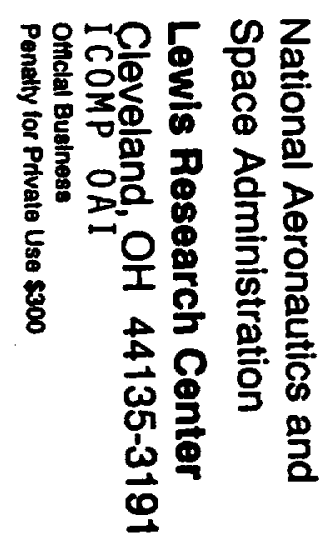

